\title{
DIGITAL DESIGN TECHNIQUES TO ASSIST IN THE COMPOSITION OF TRADITIONAL URBAN BUILDINGS
}

\author{
DOI: 10.18485/arh_pt.2020.7.ch37
}

\author{
_ James Dougherty, AICP, CNU-A, ASAI \\ Principal / Director of Design; Dover, Kohl \& Partners town planning; \\ 1571 Sunset Drive, Coral Gables, FL 33143. USA; jdougherty@doverkohl.com
}

\begin{abstract}
The design process of traditional urban fabric historically relied entirely on manual drawing techniques. Recent advances in digital design technology, including 3D modelling and light ray tracing, have unlocked several useful new techniques that can be harnessed to augment specific aspects of the design process of traditional urban building types. The paper examines 3 primary methods:

1. Seamless movement between orthographic and perspective views. Traditional designs strive for logical plan arrangements which, once built, are experienced in three-dimensions. Advances in digital modelling technology now allow designs to be quickly modelled and then easily viewed from a variety of angles, for improved evaluation of proposed forms during the iterative development of design ideas.
\end{abstract}

2. Accurate simulation of shadow shapes and light effects. Traditional urban architecture depends on careful composition of shadow shapes to expressively subdivide and articulate building masses. Digital 3D modelling and light ray tracing software allow building mouldings, projections and other sculptural forms to be quickly and efficiently modelled and instantly tested accurately in a variety of simulated lighting conditions, improving visual feedback in the design process to facilitate more rapid and effective design iterations.

3. Visualization of design proposals at a variety of distances. Traditional urban buildings are specifically designed to be viewed from a number of different distances. Smaller order compositions are nested within larger order compositions. Larger order compositions are designed to be viewed from greater distances, smaller order compositions from closer distances. Advances in digital 3D modelling and light ray tracing software allow views of designs to be accurately simulated at a variety of distances, thus helping to facilitate well-composed nested compositions.

The paper then examines a variety of potential disadvantages of current digital design platforms in the design of traditional urban buildings and concludes with suggestions for how they can be rectified to improve future outcomes.

KEYWORDS _ digital design, traditional urban architecture

\section{INTRODUCTION}

The design process of traditional urban fabric historically relied entirely on manual drawing techniques. While these methods have resulted in countless sublime built historic urban ensembles throughout the world, recent advances in digital design technology have unlocked several useful new techniques, including 3D modelling and light ray tracing, that can be harnessed to augment specific aspects of the design process of traditional urban building types today. 


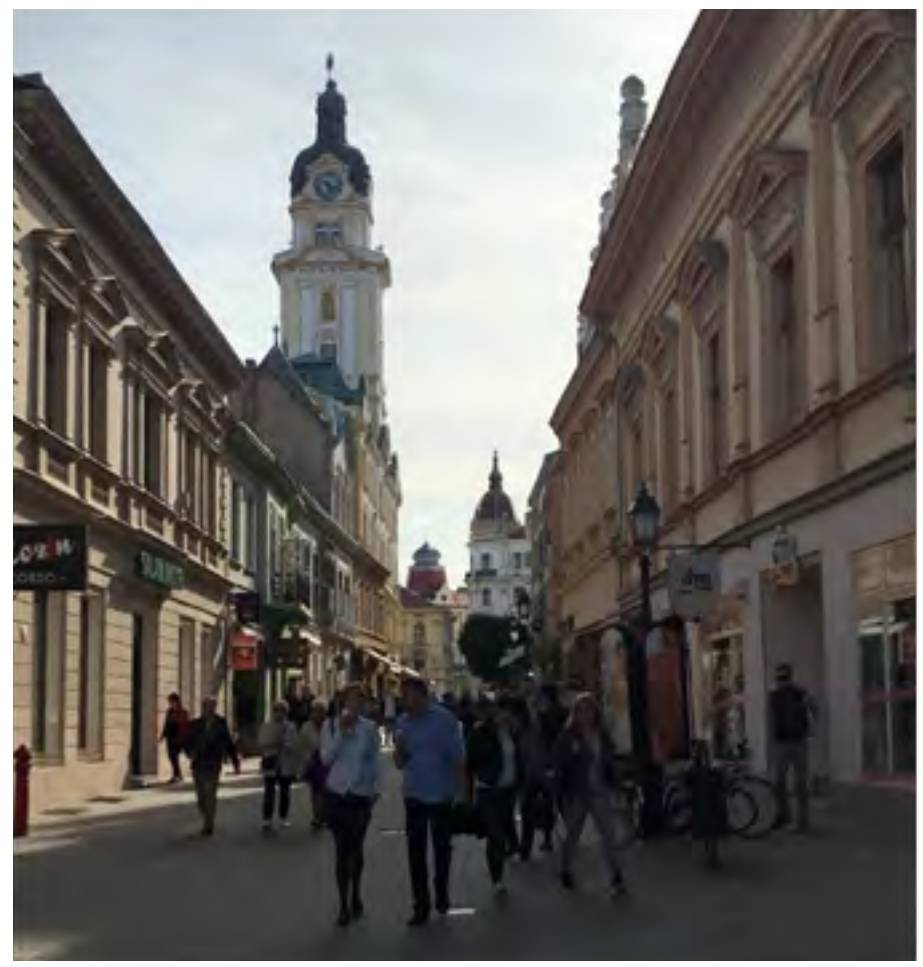

_ Figure 1: Historic urbanism such as Király utca in the historic center of Pécs, Hungary was designed using manual drawing techniques. New digital design tools can be harnessed to assist this process. (Dougherty 2020).

Three areas where new digital design tools are proving especially helpful in supplementing manual drawing methods during the design of traditional buildings are: the seamless movement between orthographic and perspective views, the accurate simulation of shadow shapes and light effects, and the visualization of design proposals at a variety of distances.

Designers should be mindful that the evolution of digital design tools, in their current state, also presents several potential disadvantages for the design of traditional urban buildings that must be managed. These include: the difficulty of performing the technique of graphic indication, the excessive ease of mechanical repetition, the predisposition for excessively flawless representation of architectonic forms, reduction of the direct connection between designer and composition due to the complexity of the interface, reduction of the constant critical attention of the human designer inherent to manual delineation, distractingly seductive photo-real entourage and lighting effects, and the restrictive optimization of digital design platforms toward drawing particular families of shapes. With a continued evolution of digital design platforms and the techniques for using them, these disadvantages can be mitigated. Harnessing the powerful advantages of digital drawing platforms, while reducing their current disadvantages, can result in enhanced capability for the design of traditional urban buildings in the future.

\section{WHY IS IT USEFUL TO DESIGN TRADITIONAL URBAN BUILDINGS TODAY?}

Traditional urbanism with its inherently compact, walkable format is useful to help manage numerous pressing problems caused by the global proliferation of suburban sprawl, including: release of 
excessive greenhouse gasses into the atmosphere through the burning of fossil fuels, accelerating loss of natural habitats and farmland due to rapid urban expansion, and a variety of human health problems resulting from the increasingly sedentary lifestyles of auto-centric land development patterns. (UN-Habitat p.87).

To achieve the construction of effective new traditional walkable urbanism it is beneficial to integrate the architectural traditions of a given region, as these usually embody evolved refinement incorporating locally calibrated reactions to climate and cultural patterns. The Congress for the New Urbanism, one of the world's preeminent organizations focusing on strategies for the reconstitution of walkable places, states in its Charter that "the development and redevelopment of towns and cities should respect historical patterns, precedents, and boundaries" and that "architecture and landscape design should grow from local climate, topography, history, and building practice". (Congress for the New Urbanism).

As traditional architecture can be useful in the design of new traditional walkable urbanism, it is therefore constructive to examine how today's new digital design tools can be harnessed to best effect to aid rather than stymie the effective design of traditional architecture.

\section{HISTORIC METHODS FOR DESIGNING TRADITIONAL BUILDINGS}

Historic methods for designing traditional buildings, particularly as formalized by the academies of Europe and America until the mid-twentieth century, are well-documented in the instructional literature of the time, such as John Harbeson's The Study of Architectural Design (1926). According to Harbeson, design should progress via iterative loops where, multiple initial design versions are explored in parallel and visually compared side-by-side. (Harbeson p.11). Positive design elements are combined, and weaker attributes discarded until a truly strong final design solution is achieved. As designs advance, they proceed from lower resolution to higher resolution in a series of nested designs. (Harbeson p.15). Early design iterations focus on the fundamental parti while details are communicated in an intentionally vague manner using a technique of graphic shorthand termed "indication". (Varon p.43). A direct physiological connection links the mind, eyes and hands of the designer with the drawing tools and composition being developed. (Curtis p.174). The traditional architectural design process is integral to the content of the architectural compositions produced, and modifications are therefore most effectively introduced with awareness of the consequences.

\section{WHAT ARE ADVANTAGES OF NEW DIGITAL TECHNIQUES IN THE DESIGN OF TRADITIONAL URBAN BUILDINGS?}

\section{Seamless moevement between ortographic and perspective views}

Architects express their designs through the orthographic views of plan, elevation, and section to explore the composition of building elements and their relationship to one-another. (Curtis p.161). Architects know that such orthographic drawings are conventions, and that built work will invariably be experienced three-dimensionally within its urban context. (Curtis p.141). Whenever possible, architects historically built physical models to aid three-dimensional visualization, but when this was not possible they were left to only imagine what their designs might look like in the round and, after construction, to save lessons learned for future efforts. (Varon p.19). Three-dimensional hand drawing, while frequently performed, was invariably viewed with skepticism regarding its accuracy. (Magonigle p.xv).

Today by contrast, user-friendly digital modelling tools have made quick and accurate three-dimensional exploration and testing of forms during the architectural design process widely accessible to designers. 

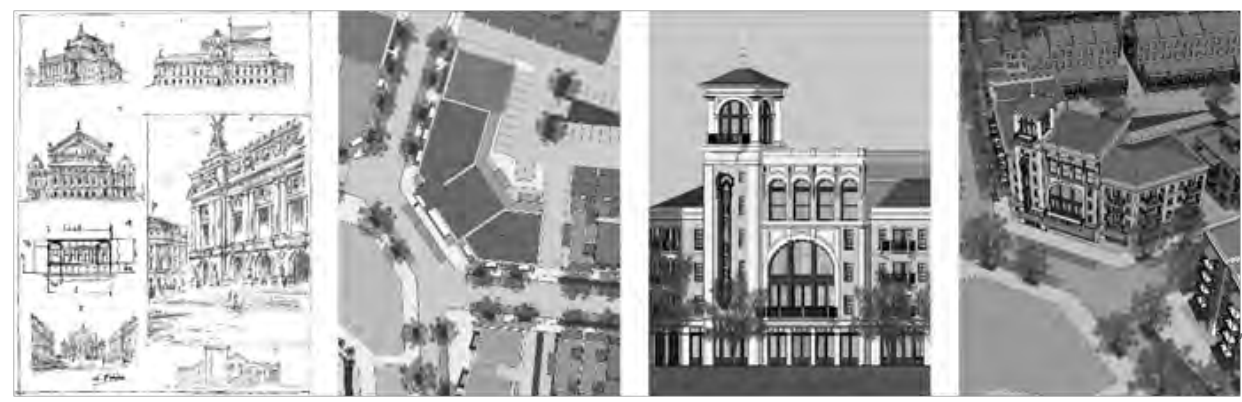

_ Figures 3, 4 and 5 (right): Design study of a new focal building, in orthographic and perspective views, utilizing digital modelling software. (Dover, Kohl \& Partners 2020)

It is now possible for a designer to view a composition not just in traditional orthographic views but to effortlessly rotate it in virtual space to be viewed from any angle imaginable. Modern digital modelling techniques can therefore be harnessed to produce traditional urban buildings that have been accurately vetted from the three-dimensional view angles likely to be experienced by end users, an aid to the achievement of satisfying built results not available to historic architects. (Claypool 4. Early Digital Explorations). A notable vulnerability of this new capability requiring management, however, is that designs can be advanced pictorially without adequate attention spent on development of the composition of plan, elevation and section. (Magonigle p.133).

\section{Accurate simulation of shadow and light effects}
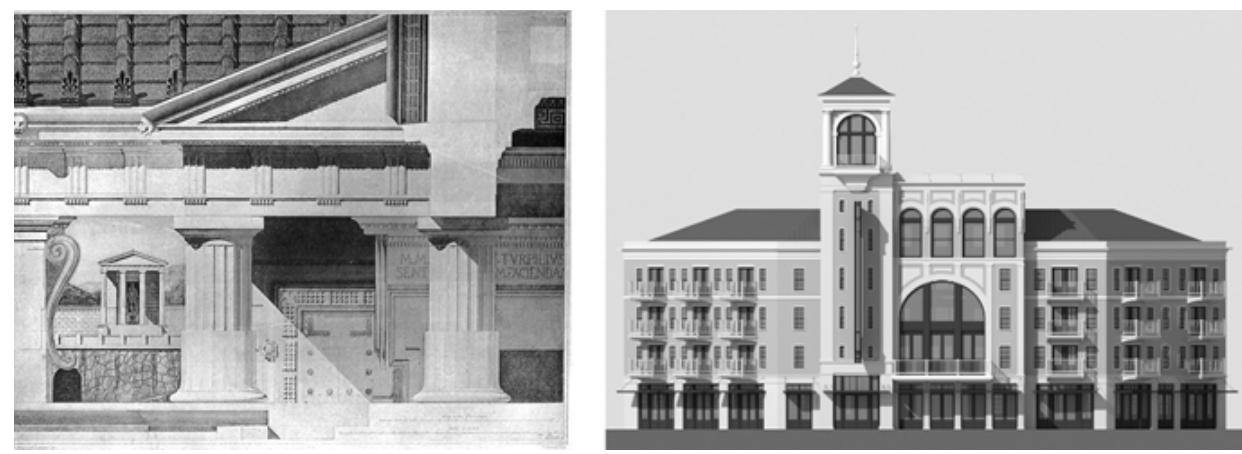

_ Figure 6 (left): 1905 drawing depicting a restored Temple of Hercules at Cori demonstrating Beaux-Arts conventions for rendered shades and shadows. (d'Espouy, Plate C).

_ Figure 7 (right): Elevation study of a new focal building with shadows cast according to Beaux-Arts conventions utilizing digital modelling and ray tracing software. (Dover, Kohl \& Partners 2020).

Traditional urban architecture is designed to be seen in real world lighting conditions. Shapes of shadows cast by direct sunlight falling across architectonic forms have evolved over many generations to enhance a building's contribution to legibility of the urban environment while helping to form a building's particular visual identity. (Robinson p.27). The Beaux-Arts convention of casting shadows from the upper left to lower right at an angle of 45 degrees made it possible to accurately gauge three-dimensional depth of architectural elements within a two-dimensional drawing. (Harbeson p.55). As this convention was adhered to by all practitioners, it also made very accurate side-by-side comparisons of competing design proposals possible. (Curtis p.134). Traditionally, cast shadow shapes were quickly estimated by eye in early freehand sketches and only later painstakingly manually projected on orthographic drawings. They were then rendered in graded tones to represent the 
effects of direct, ambient, reflected, and occluded light as accurately as possible. (Curtis p.134). The slow manual work required to project and render accurate shades and shadows, while providing an important opportunity to apply the designer's artistic judgement, limited the ability to draw and accurately test multiple design iterations quickly. (Harbeson p.55). Today, with digital lighting software, architectural forms and shadow shapes can easily be tested accurately throughout the entire design process in realistically simulated lighting conditions. (Claypool 7. Computing Nature). This capability, unavailable to historic designers, can improve and expedite the process of visually vetting design iterations. A potential drawback of this automated casting of shadow shapes, however, can be a reduction of the focused critical attention of the skilled human designer to this key compositional element. (Curtis p.172).

\section{VISUALIZATION OF DESIGN PROPOSALS AT A VARIETY OF DISTANCES}

Traditional urban buildings were designed to enhance the viewer's experience as they moved through the city and were therefore designed to be viewed from a variety of distances. (Curtis p.119). Traditionally, overall massing and silhouette, enhanced by major elements such as cornice shadows, were meant to be legible from great distances. At closer distances, successively finer-grained layers of composition reveal themselves. Upon approach, the rhythm of structural center lines and the pattern of openings become visible. Closer still and the relative proportions of columns and entablatures integrated into the façade become visible. The finest articulation of sculptural detail is often not visible until directly in front of a building. This successive revealing of layers of nested compositional articulation is a primary reason why movement through traditional urban sequences comprised of such buildings is such a satisfying experience. (Varon pp. 22, 23, Plate XVII). Traditional architects therefore employed a design process that allowed them to focus on and refine each nested layer of design sequentially. First, sketches are drawn at a very small scale, so that the entire façade is visible on the drawing board before the designer at a glance, and only large order compositional elements are drawn with precision. Smaller order elements, if shown at all, are merely indicated with a quick graphic shorthand. (Curtis pp.174, 176) Once the large order composition reaches a satisfying level of resolution, the designer proceeds to the next level of nested compositional elements by manually redrawing at a larger scale successively until finally all nested layers are developed and refined. (Curtis p.196).
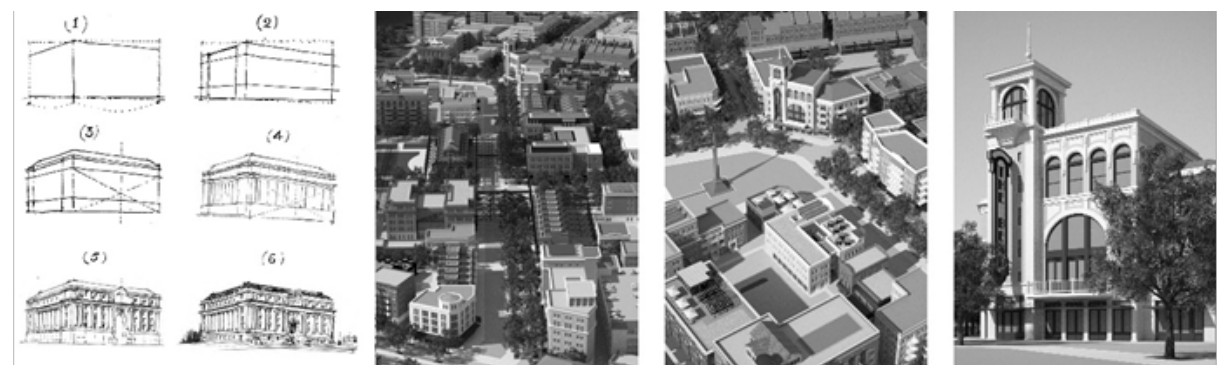

_ Figure 8 (left): Layers of traditional nested composition. (Varon, Plate XXII).

_ Figures 9, 10 and 11 (right): Examination of a new focal building design in its urban context from multiple distances and viewpoints utilizing digital modelling and ray tracing software. (Dover, Kohl \& Partners 2020).

Today, digital drawing and modelling tools make viewing a draft composition at multiple scales very easy to accomplish. It is often taken for granted that today one need only 'zoom out' to focus on large order compositional elements and 'zoom in' as each successive layer of design is refined. (Claypool 4. Early Digital Explorations). This quite common capability of today's digital drawing plat- 
forms would have been unspeakably helpful to historic designers of traditional urban buildings. A potential liability of this new digital capability, however, is the ease of prematurely proceeding to the design of smaller order compositions before larger order compositions are adequately resolved. (Varon p.43).

\section{WHAT ARE DISADVANTAGES OF CURRENT DIGITAL DESIGN TECHNIQUES?}

While today's digital design tools offer the previous potential, though qualified, advantages; they can also present several potential obstacles to the design of traditional urban buildings:

\section{The difficulty of performing the technique of graphic indication}

Digital design platforms are optimized for precise delineation of forms and suffer from an inability to draw vaguely. (Claypool 4. Early Digital Explorations). The difficulty of utilizing the technique of graphic indication when designing digitally is rooted in both hardware and software causes. Regarding hardware, the typical interface with digital design technology is the mouse and keyboard. The mouse is optimized for small, precise movements of the hand and not for the large, gestural movements of traditional freehand pencil drawing. The keyboard is an even further interruption of the traditionally direct link from designer's mind, to hand, to paper and it reduces the ability to generate a seamless physiological connection between the designer and the work being produced. (Varon p.19). Additionally, digital design software has focused on facilitating the drawing of precise, vector-based shapes. (Claypool 7. Computing Nature). The very precision of these shapes is antithetical to the vague and suggestive character of the indication so critical to quick and efficient early visualization of the complex nested design ideas of traditional architecture. (Varon p.23).

\section{The excessive ease of mechanical repetition}

When all forms are drawn by hand, repetition beyond a certain point becomes physically and mentally fatiguing to the designer and increases the urge to incorporate variation within a design. (Varon p.29). The ease of 'copy / paste' and 'array' functions of today's digital design platforms allow mechanical repetition of elements far beyond what would have been tolerable to a traditional designer employing hand drawing. (Claypool 4. Early Digital Explorations). This ease of repetition can result in the skewing of the judgement of the designer, and adversely affect the character of the designs produced (Varon p.29).

\section{The predisposition for excessively flawless representation of architectonic forms}

Traditional hand drawing techniques feature a degree of variability and imperfection even when executed by a highly skilled practitioner. This natural variability is helpful in conveying the real natural imperfection of built architecture, particularly under the effects of weathering, gravity and time. (Harbeson p.48). Digital drawing tools, by contrast, make possible the representation of lines, tones and gradations with a level of superhuman precision that is not physically achievable in hand drawing. (Claypool 4. Early Digital Explorations). This can result in a lack of simulation of the inevitable variability of weathered and aged forms in the real world which can inadvertently cause designers to select forms that embody a high level of platonic perfection but which can suffer inordinately when exposed over time to the real world rigors of weathering and gravity. (Harbeson p.48).

\section{Reduction of the constant critical attention of the human designer inherent to manual delineation}

A goal of evolving digital design platforms has been to insert automated computational tools into the form-making decision process. (Claypool 8. Parametric Explosion). In contrast, traditional hand drawing techniques require the engagement of the physiology and consciousness of the designer with every millimeter of every line being drawn. (Varon p.19). This can lead to different design decisions than when lines are instead generated by a computer. For instance, in BIM and parametric 
drawing systems a design can be produced via numerical inputs then a three-dimensional representation of the design is automatically generated by the computer software. This three-dimensional representation is inherently subject to less precise scrutiny than if it were hand-drawn by a skilled human practitioner and can result in unintended and potentially undesirable outcomes. (Claypool 7. Computing Nature).

\section{Distractingly seductive photo-real entourage and lighting effects}

Digital modelling and rendering software have achieved an extraordinarily sophisticated level of representation of trees, vehicles, people and other entourage items and simulation of various lighting conditions. These advances have made it possible to place a proposed architectural composition into a graphic setting that is so visually seductive, that objective judgment of the merits of the architectural design being represented can be hampered. (Harbeson p.129).

\section{The resctrictive optimization of digital design platforms toward drawing particular families of shapes}

When a drawing platform makes representation of a certain family of forms easier or more difficult to draw, this inevitably influences the design choices made by designers using these platforms. Traditional hand drawing techniques feature the ability to seamlessly transition between lines drawn with mechanical instruments and lines drawn freehand. The effect of this flexibility can be seen in the frequent combination of straight lines and organic, sculptural shapes within traditional architectural compositions. (Harbeson p.48). By contrast for example, orthogonal and rectilinear forms have, for example, long been easier to draw with digital design software than complex curvilinear forms. (Claypool 4. Early Digital Explorations). While many advances have been made in recent years in the ability to draw organic forms with digital software platforms, they still commonly lack the ability to easily produce the nuanced and controlled contour lines commonly found in the representation of traditional figurative architectural sculpture. (Harbeson p.48).

\section{CONCLUSIONS}

Advances in digital design technology, including 3D modelling and light ray tracing, can be harnessed to augment specific steps in the design of traditional urban building types. From the seamless movement between orthographic and perspective views, to the accurate simulation of shadow shapes and light effects, to the visualization of design proposals at a variety of distances; the capabilities of digital design technology can be utilized to enhance and expedite traditional manual design methods.

It is hoped that a careful and reasoned integration of new digital capabilities into time-tested manual design workflows may continue to prove beneficial in the future service of those designing traditional urban building types. The following are proposals for ways that traditional and digital methods can be further integrated to better serve traditional design methods:

\section{Integrate true manual drafting tools with digital platforms}

A hardware and software evolution that would allow a designer to utilize true manual drafting instruments to interface with digital design software would be extremely helpful by allowing the digital design process to mimic traditional techniques as closely as possible. Among the positive impacts would be: improvement of the directness of connection between designer and design content during the design process, reduction of excessive ease of mechanical repetition, and facilitation of seamless alternation between orthogonal and freeform line geometries. To achieve these benefits, digital stylus technology should also continue to progress to achieve the true precision and responsiveness of a manual drafting pencil. 


\section{More seamlessly integrate hand-drawn indication with digital workflows}

The use of a digital stylus to replicate the soft pencil needed to hand draw the intentionally vague lines critical for the technique of indication is already possible with recent advances in stylus technology. This could be further facilitated by pairing it with software advances that allow toggling to pre-set standardized drawing scales, and which render intuitive the progression from vaguely indicated lines to precisely defined vector graphics. This would function best if combined with a tablet screen that is large enough to replicate the size of a traditional drawing sheet, so that a design drawn at a standardized scale could be seen and worked on in its entirety without the need for constant zooming in and out.

\section{Facilitate beaux-arts delineation conventions in software pre-sets}

In order to counteract the tendency of overly-seductive entourage and lighting effects to reduce the ability to critically assess a design on its own architectural merits, digital design platforms could facilitate more easily presenting an architectural design utilizing the Beaux-Arts convention of linework plan, elevations and section drawn at a set conventional scale, and rendered in grayscale or reduced chroma with shadows cast down and to the right at an angle of 45 degrees. Ready access in a default setting to this conventional presentation graphic technique would make it possible to view multiple designs in a graphically consistent way and would therefore facilitate more reliable comparative evaluation of design content.

\section{Evolve controlled imperfection in delineation pre-sets}

To counteract the tendency of digital rendering software to present designs with an unrealistic degree of perfection, additional evolution of digital design platforms should be undertaken to enhance the ability to easily render designs with the simulated effects of time, gravity and weathering.

\section{REFERENCES:}

_ Claypool, Mollie. (2020). The Digital in Architecture: Then, Now and in the Future. Space 10.

- Congress for the New Urbanism. (1996). The Charter of the New Urbanism. CNU, Washington DC.

- Curtis, Nathaniel Cortland. (1923). Architectural Composition. J H Hansen, Cleveland Ohio.

- d'Espouy, Hector. (1905). Greek and Roman Architecture in Classic Drawings. Classical America. Dover

Publications, New York.

- Harbeson, John F. (1926). The Study of Architectural Design. W. W. Norton \& Company, NY

- Magonigle, H. Van Buren. (1926). Architectural Rendering in Wash. Charles Scribner's Sons, NY

- Robinson, John Beverley. (1908). Architectural Composition. D Von Nostrand Company / B T Batsford

/ 94 High Holborn, New York / London.

- Varon, David J. (1916). Indication in Architectural Design. The William T Comstock Company, NY.

- UN-Habitat. (2016). Urbanization and Development: Emerging Futures. United Nations Human Settle-

ments Programme. 